\title{
Flux Growth of Phosphide and Arsenide Crystals
}

\author{
Jian Wang ${ }^{1 * t}$, Philip Yox ${ }^{2,3}$ and Kirill Kovnir ${ }^{2,3 *+}$ \\ ${ }^{1}$ Department of Chemistry, Wichita State University, Wichita, KS, United States, ${ }^{2}$ Department of Chemistry, lowa State \\ University, Ames, IA, United States, ${ }^{3}$ Ames Laboratory, U.S. Department of Energy, Ames, IA, United States
}

Flux crystal growth has been widely applied to explore new phases and grow crystals of emerging materials. To accommodate the needs of high-quality single crystals, the flux crystal growth should be reliable, controllable, and predictable. The selections of suitable flux and growth conditions remain empirical due to the lack of systematic investigation especially for reactions, which involve highly volatile components, such as $P$ and As. Considering the flux elements, often the system in question is a quaternary or

OPEN ACCESS

Edited by:

Hans-Conrad zur Loye,

University of South Carolina,

United States

Reviewed by:

Dirk Johrendt

Ludwig Maximilian University of

Munich, Germany

Arthur Mar,

University of Alberta, Canada

*Correspondence:

Jian Wang

jian.wang@wichita.edu

Kirill Kovnir

kovnir@iastate.edu

TORCID:

Jian Wang

orcid.org/0000-0003-1326-4470

Kirill Kovnir

orcid.org/0000-0003-1152-1912

Specialty section:

This article was submitted to

Inorganic Chemistry,

a section of the journal

Frontiers in Chemistry

Received: 14 December 2019

Accepted: 28 February 2020

Published: 02 April 2020

Citation:

Wang J, Yox P and Kovnir K (2020)

Flux Growth of Phosphide and

Arsenide Crystals.

Front. Chem. 8:186.

doi: 10.3389/fchem.2020.00186 a higher multinary system, which drastically increases complexity. In this manuscript, on the examples of flux growth of phosphides and arsenides, guidelines of flux selections, existing challenges, and future directions are discussed. We expect that the field will be further developed by applying in situ techniques and computational modeling of the nucleation and growth kinetics. Additionally, leveraging variables other than temperature, such as applied pressure, will make flux growth a more powerful tool in the future.

Keywords: flux, crystal growth, phosphide, arsenide, salt flux, metal flux, self-flux

\section{INTRODUCTION}

The fundamental research goals of solid state chemistry, materials science, and condensed matter physics are to establish correlations between crystal structure and physical properties (Pamplin, 1980; Kanatzidis et al., 2005; The National Academies of Sciences Engineering Medicine, 2009). The single crystalline solids provide a suitable and simple platform due to the absence of grain boundaries and suppression and/or control of defects. Large, mm-sized or even bigger single crystals are indispensable for the characterization of anisotropic physical properties, such as magnetic, heat and charge transport, or optical properties (Phelan et al., 2012; Babu et al., 2018; Liu et al., 2018; Canfield, 2020). The scientific community has raised high standards for single crystal growth. Growth of single crystals should be fast, controllable, and capable of handling complex systems (Pamplin, 1980; Kanatzidis et al., 2005; The National Academies of Sciences Engineering Medicine, 2009; Canfield, 2020). One of the options is to grow crystals from a high-temperature molten media, flux, which is widely used for growth of different intermetallic, semiconducting, and insulating compounds of diverse chemical nature ranging from oxides and halides to metal alloys (Pamplin, 1980; Canfield and Fisk, 1991; Kanatzidis et al., 2005; Bugaris and zur Loye, 2012; Phelan et al., 2012; Juillerat et al., 2019; Canfield, 2020). The art of flux crystal growth is a combination of science and technology. The science part requires comprehensive knowledge of chemical bonding and reactivity of components and flux, combined with knowledge of the thermodynamics and kinetics of the growth processes. The technology aspect of the crystal growth is in its "trial and error" nature, which requires multiple attempts sometimes guided by the grower's intuition. In situ studies of the flux growth have provided insights regarding the structure of the liquid phase and cascades of solid-solid transformation occurring during heating and cooling (Shoemaker et al., 2014; Woo et al., 2018). 
Several general aspects of flux selection, together with examples and challenges, are discussed in this paper with focus on ternary and multinary phosphides and arsenides. The chemistry of phosphides and arsenides at elevated temperature is complicated because of the high vapor pressure of $\mathrm{P}$ or As. The volatility of $\mathrm{P}$ and As has resulted in poor exploration of many metal-phosphorus (arsenic) phase diagrams in the areas of high pnictogen content. Nevertheless, compounds with substantial $\mathrm{P}(\mathrm{As})$ content often exhibit interesting chemistry and useful practical properties, with potential applications as heat conductors, thermoelectrics, catalysts, and non-linear optical materials (Soheilnia et al., 2007; Lindsay et al., 2013; Dolyniuk et al., 2017; Nuss et al., 2017; Pöhls et al., 2017; Li et al., 2018; Owens-Baird et al., 2018, 2019; Woo et al., 2018; Coleman et al., 2019; Mark et al., 2019; Yu et al., 2019).

\section{Flux: General Considerations}

Various options exist for flux growth of inorganic crystals, such as metal flux, salt flux, or self-flux. Basic considerations for flux choice involve three aspects: solubility of reactants in flux, reaction path, and nucleation and growth of the target crystals (Figure 1, bottom). The presumptions about solubility of reactants in the flux are mainly drawn from the binary phase diagrams (Figure 1G). The flux crystal growth process is usually accompanied by chemical reaction, distinguishing itself from the growth of molecular compounds where recrystallization techniques prevail. In most cases, the flux plays a dual role in the crystal growth process: facilitating chemical reactions and aiding crystal growth (Figures $\mathbf{1 H}, \mathbf{I}$ ). In addition to the chemical and physical properties of the reactants and the products, interactions between the reactants and the flux should also be considered. The controllable nucleation and growth for a new system are challenging to achieve, due to the lack of systematic investigations. To compensate, a series of experiments are performed screening several flux/reagent ratios and heating/cooling rates. Owing to the limited size of this minireview, the comprehensive discussions of thermodynamics and kinetics are not covered here.

For exploring a new system, selecting a suitable flux is crucial. The following considerations of the flux properties are important:

\section{Solubility}

Moderate solubility of the target phase in the flux and a lowtemperature dependence of the solubility are required. Low solubility of the reactants and the target phase will prevent chemical reactions and crystal growth. However, very high solubility will result in unsaturated solutions inhibiting the crystallization of the target phase. The temperature dependence of a target phase's solubility plays a critical role in the crystal growth process because it determines the cooling rate. For a new system or a new compound, solubility and its temperature dependence are often unknown and not easy to determine, but a few hints may be obtained by running several model experiments. Cooling rates as low as $0.4 \mathrm{~K} / \mathrm{h}$ have been used, requiring weeks to complete the growth.

\section{Inertness}

The inertness of flux is required to avoid formation of stable phases between the flux and the reactants. Forming fluxcontaining compounds will shift the stoichiometry and disturb the nucleation process. Another aspect of flux inertness is undesirable doping of the target phase with flux components.

\section{Melting Temperature}

Large temperature ranges are ideal for crystal growth. Favorable fluxes have low melting temperatures coupled with high boiling points.

\section{Toxicity}

Non-toxic flux with low vapor pressure is safer and easier to handle for the environment and researchers. For example, $\mathrm{Hg}$ is rarely used as flux.

\section{Container}

To avoid incorporation of the container elements into the system and/or the target crystals, chemical reactions between flux and container are highly undesirable. If the flux has low solubility of volatile reactants ( $\mathrm{P}, \mathrm{As})$ or the flux itself has a high vapor pressure, the container volume is another parameter that must be considered.

\section{Removal}

Flux should be removed after the growth is complete. Several methods including mechanical separation and polishing of crystals, high-temperature centrifugation of liquid flux, and dissolving flux at or near room temperature are frequently used. In the latter case the flux nature defines whether simple solvents (water, ethanol) or acid ( $\mathrm{HCl}, \mathrm{H}_{2} \mathrm{O}_{2}$ /acetic acid) should be used. Dissolution requires the target phase to be stable in that media, limiting the choice of fluxes. Thus, $\mathrm{KCl} / \mathrm{NaCl}$ is preferred over $\mathrm{Sn}$ flux for acid-sensitive materials due to the solubility of chlorides in water. $\mathrm{Bi}$ and $\mathrm{Pb}$ fluxes are not soluble in non-oxidizing acids and require the presence of hydrogen peroxide in the dissolving media.

\section{Price}

Flux is used in high excess in comparison to amounts of target materials or reactants. Thus, very expensive fluxes are always not the first choice especially with "trial and error" approaches. For example, $\mathrm{Au} / \mathrm{Si}$ eutectic $\left(\mathrm{T}_{\text {melting }}=636 \mathrm{~K}\right)$ might be a good flux, but it is rarely used due to the high price of gold.

\section{Viscosity, Density, and Polarity}

The high viscosity of flux may result in non-stationary mass transport, which brings no benefits to crystal growth. The densities of reactants and the flux should be comparable. Analogous to mixing water and benzene in a beaker, a phase separation may occur during flux crystal growth. In water/benzene combination not only density but also polarity of the liquids plays an important role. For flux synthesis, the rule of thumb is that metallic phases grow better from metallic fluxes $(\mathrm{Sn}, \mathrm{Pb}, \mathrm{Bi})$, while semiconducting crystals are easier to grow from semiconducting fluxes (salts). This is not a strict rule, and exceptions are known. 


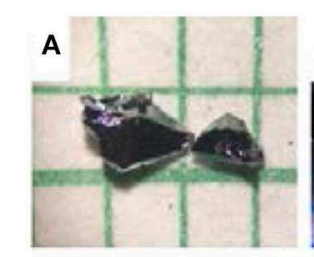

$\mathrm{BaCu}_{2} \mathrm{P}_{4}$ in $\mathrm{Sn}$

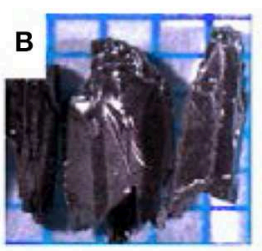

$\mathrm{Ba}_{3} \mathrm{Cd}_{2} \mathrm{As}_{4}$ in $\mathrm{Pb}$

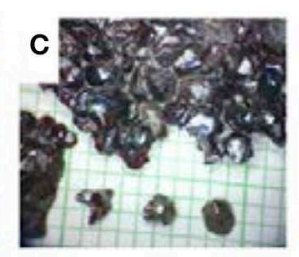

$\mathrm{Mg}_{3} \mathrm{Si}_{6} \mathrm{As}_{8}$ in $\mathrm{Bi}$
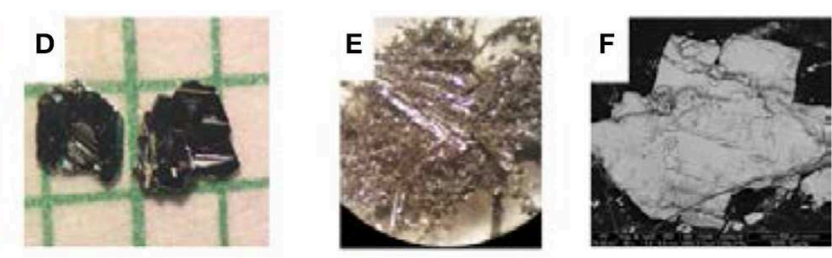

$\mathrm{CeCd}_{3} \mathrm{P}_{3}$ in $\mathrm{KCl} / \mathrm{NaCl} \mathrm{CsZn}_{0.5} \mathrm{Si}_{5.5} \mathrm{As}_{12}$ in $\mathrm{CsCl} \quad \mathrm{RbFe}_{2} \mathrm{As}_{2}$ in $\mathrm{FeAs}$

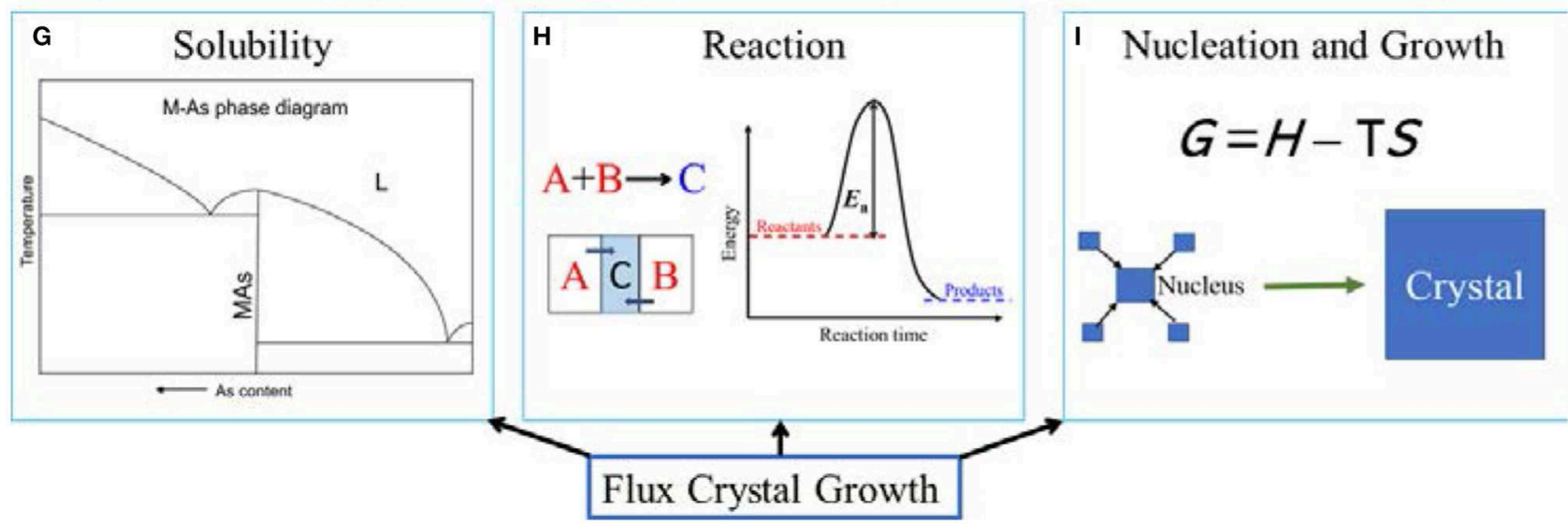

FIGURE 1 | Top: Examples of phosphide and arsenide crystals grown in different fluxes (Wang et al., 2013; Dolyniuk et al., 2015; Woo et al., 2018, 2019a). (D,F) show unpublished crystals synthesized in Kovnir group. Bottom: main aspects of flux crystal growth. (A) $\mathrm{BaCu}_{2} \mathrm{P}_{4}$ in $\mathrm{Sn}_{2}$ (B) $\mathrm{Ba}_{3} \mathrm{Cd}_{2} \mathrm{As}_{4}$ in $\mathrm{Pb}_{\text {, (C) }} \mathrm{Mg}_{3} \mathrm{Si}_{6} \mathrm{As}_{8}$ in $\mathrm{Bi}_{\text {, }}$ (D) $\mathrm{CeCd}_{3} \mathrm{P}_{3}$ inKCl/ $\mathrm{NaCl}$, (E) $\mathrm{CsZn}_{0.5} \mathrm{Si}_{5.5} \mathrm{As}_{12}$ in $\mathrm{CsCl}$, (F) $\mathrm{RbFe}_{2} \mathrm{As}_{2}$ in FeAs, (G) M-As phase diagram, (H) Solid-state reaction, (I) Nucleation and growth.

The bottom line is that no single flux can satisfy all the criteria. However, these guiding principles can help to make rational flux choice for synthesis.

\section{Metal Flux Examples}

Phosphorus and arsenic can form binary pnictides with almost all metals (excluding $\mathrm{Hg}$ and $\mathrm{Bi}$ ) (Shatruk, 2019). Mercury has high vapor pressure and is toxic, thus bismuth should be the first choice for flux to grow phosphides and arsenides based on the inertness of flux criteria. Bi works well for arsenides due to the relatively high solubility of As in Bi. However, the solubility of $\mathrm{P}$ in $\mathrm{Bi}$ is quite low, and $\mathrm{Sn}$ flux is preferred for phosphides. Despite the existence of a number of binary Sn$\mathrm{P}$ phases, multiple complex phosphides have been grown from Sn flux (Kanatzidis et al., 2005). Tin phosphides can be washed away with acid and have moderate melting points below $850 \mathrm{~K}$. Binary tin arsenides have similar melting points to tin phosphides of $\sim 870 \mathrm{~K}$; however, $\mathrm{Sn}$ has somewhat limited success as a flux to grow arsenides; see example of $\mathrm{RCo}_{2} \mathrm{Pn}_{2}$ phases below. A common side-product of arsenide growth attempts in tin flux is $\mathrm{SnAs}$ binary with $\mathrm{NaCl}$ structure, which is different from SnP. The latter is a metastable phase with a unique crystal structure (Gullman, 1990). More systematic studies are required to figure out whether SnAs is an important intermediate, which affects crystallization processes or just a common side-product.

$\mathrm{Pb}$ was recently reported to form binary $\mathrm{PbP}_{7}$ (Schäfer et al., 2014), which demonstrates higher affinity of $\mathrm{Pb}$ to $\mathrm{P}$ compared to $\mathrm{Bi}$, despite the exact phase diagram for the $\mathrm{Pb}-\mathrm{P}$ system was not reported. Svilen Bobev et al. reported multiple successful crystal growths of both phosphides and arsenides from lead flux (Bobev et al., 2009; Saparov and Bobev, 2010; He et al., 2016). Finally, other metallic fluxes, such as $\mathrm{Zn}, \mathrm{Al}, \mathrm{Ga}$, and In have been used for selected cases of phosphide and arsenide crystal growth.

\section{$\mathrm{BaCu}_{2} \mathrm{P}_{4}$ in Sn Flux}

Synthesis of twisted clathrate $\mathrm{BaCu}_{2} \mathrm{P}_{4}$ (Dolyniuk et al., 2015) is hampered by the existence of another clathrate with close composition, $\mathrm{BaCu}_{2} \mathrm{P}_{3.75}$. Thermal analysis confirmed that $\mathrm{BaCu}_{2} \mathrm{P}_{4}$ converts into $\mathrm{BaCu}_{2} \mathrm{P}_{3.75}$ upon heating prior to melting, which explains the experimental observations that high-temperature syntheses of $\mathrm{BaCu}_{2} \mathrm{P}_{4}$ always resulted in the formation of $\mathrm{BaCu}_{2} \mathrm{P}_{3.75}$. In turn, $\mathrm{Ba}$ and $\mathrm{Cu}$ starting materials are not active at low temperatures $(<1,000 \mathrm{~K})$ due to their high melting points, 1,003 and $1,353 \mathrm{~K}$, respectively. To resolve this issue, flux growth was applied. $\mathrm{P}$ has high solubility in Sn (Zavrazhnov et al., 2018), which can be removed by centrifugation. The mm-sized $\mathrm{BaCu}_{2} \mathrm{P}_{4}$ crystals were successfully grown by Sn flux at 1,073 K (Figure 1A).

\section{$\mathrm{Mg}_{3} \mathrm{Si}_{6} \mathrm{As}_{8}$ in Bi Flux}

The Mg-Si-As ternary system has been overlooked for many years with only one theoretically predicted compound $\mathrm{MgSiAs}_{2}$ (Woo et al., 2018). Our synthetic efforts discovered three new ternary compounds, $\mathrm{MgSiAs}_{2}, \mathrm{Mg}_{3} \mathrm{Si}_{6} \mathrm{As}_{8}$, and $\mathrm{Mg}_{3} \mathrm{Si}_{3} \mathrm{As}_{8}$ (Woo et al., 2018; Wang et al., 2019). MgSiAs, was confirmed to have chalcopyrite structure type, while the other two compounds crystallize in new structure types. The crystal growth of the $\mathrm{Mg}-$ $\mathrm{Si}-\mathrm{As}$ system is challenging due to the combination of high vapor pressure of $\mathrm{Mg}$ and As and the inertness of Si at reaction 
temperatures. As discussed above, $\mathrm{Bi}$ is preferred over $\mathrm{Sn}$ flux for arsenides. Indeed, mm-sized red crystals of $\mathrm{Mg}_{3} \mathrm{Si}_{6} \mathrm{As}_{8}$ were obtained in Bi flux (Figure 1C).

\section{$\mathrm{Ba}_{3} \mathrm{Cd}_{2} \mathrm{As}_{4}$ in $\mathrm{Pb}$ Flux}

When exploring the synthesis of $\mathrm{Ba}_{3} \mathrm{Cd}_{2} \mathrm{As}_{4}$ (Wang et al., 2013), Cd and Pb flux were applied to grow crystals. Cd has a moderate range of operation between melting and boiling points, 594 and 1,038 K, respectively. Using Cd as a self-flux, the chances of incorporation of foreign elements were minimized. A disadvantage of $\mathrm{Cd}$ flux was the formation of binary admixture $\mathrm{Cd}_{3} \mathrm{As}_{2}$. Small crystals of $\mathrm{Ba}_{3} \mathrm{Cd}_{2} \mathrm{As}_{4}$ were grown from $\mathrm{Cd}$ flux with low yield. Larger crystals of $\mathrm{Ba}_{3} \mathrm{Cd}_{2} \mathrm{As}_{4}$ were grown from $\mathrm{Pb}$ flux. $\mathrm{Pb}$ has a comparable melting point and much higher boiling point than $\mathrm{Cd}, 603$ and $2,023 \mathrm{~K}$, respectively. The $\mathrm{Ba}$, $\mathrm{Cd}$, and As reactants have reasonable solubility in $\mathrm{Pb}$ at elevated temperatures. $\mathrm{Mm}$-sized crystals of $\mathrm{Ba}_{3} \mathrm{Cd}_{2} \mathrm{As}_{4}$ were grown in $\mathrm{Pb}$ flux (Figure 1B).

\section{$\mathrm{RCo}_{2} \mathrm{Pn}_{2}(\mathrm{R}=$ Rare-Earth Metal; $\mathrm{Pn}=\mathrm{P}$ and $\mathrm{As})$ in $\mathrm{Sn}$ and $\mathrm{Bi}$ Fluxes}

In the three aforementioned examples, direct reactions of elements produced the polycrystalline samples of the target phases. Often this is not the case, and flux is used to overcome high reaction barriers. For example, reactions of neat $\mathrm{La}, \mathrm{Co}$, and $\mathrm{P}$ resulted in a mixture of stable binary phosphides, LaP, and $\mathrm{CoP} / \mathrm{Co}_{2} \mathrm{P}$, which would not react with each other preventing formation of $\mathrm{La}-\mathrm{Co}-\mathrm{P}$ ternaries. Sn flux resolves these issues resulting in mm-sized crystals of $R \mathrm{Co}_{2} \mathrm{P}_{2}$, which allows for establishing of the structure-magnetic property relationships (Kovnir et al., 2010, 2011a,b; Kovnir et al., 2013; Thompson et al., 2014a; Tan et al., 2017). When switching to isostructural arsenides, tin flux failed to produce any $\mathrm{RCo}_{2} \mathrm{As}_{2}$ phases. Instead Bi flux was successfully applied (Thompson et al., 2011, 2014b; Tan et al., 2016a, 2018). Detailed structural characterizations revealed that $\mathrm{Bi}$ is capable of partially replacing $R^{3+}$ cations, probably due to its trivalent nature (Thompson et al., 2011). No evidences of $\mathrm{Sn}$ incorporation into crystals of $R \mathrm{Co}_{2} \mathrm{P}_{2}$ were found. However, divalent $\mathrm{Sn}$ was reported to partially replace $\mathrm{Ba}^{2+}$ in isostructural $A \mathrm{Fe}_{2} \mathrm{As}_{2}$ superconductors $(A=\mathrm{Ba}, \mathrm{K})$ (Mathieu and Latturner, 2009).

\section{$\mathrm{GeP}$ in $\mathrm{Sn}$ and Bi Fluxes}

Incorporation of the metal flux components into the crystal structure is one of the reasons for discarding certain fluxes. Elemental $\mathrm{Ge}$ and $\mathrm{P}$ will not react with each other at the temperatures at which binary $\mathrm{GeP}$ is stable, thus calling for flux application. The growth of GeP van-der-Waals semiconductor crystals were achieved from Sn flux (Lee et al., 2015). The detailed structural characterizations show that a significant degree of disorder in both Ge and $\mathrm{P}$ sites is introduced by partial replacement of $\mathrm{Ge}$ with larger $\mathrm{Sn}$ atoms. Sn doping resulted in drastic decrease in melting/decomposition temperature by $228 \mathrm{~K}$ compared to the pristine GeP. Similarly, over an order of magnitude increase in electrical resistivity was detected for Sndoped GeP. Undoped GeP was successfully synthesized using Bi flux instead of Sn. No incorporation of Bi was detected by SEM/EDS and crystallographic investigations (Lee et al., 2015).

\section{Salt Flux Crystal Growth Examples}

Many inorganic pnictide crystals can be grown in salt fluxes. Salt fluxes are high-temperature ionic liquids (Table 1). The salt flux can be inert $(\mathrm{NaCl} / \mathrm{KCl})$ or reactive $\left(\mathrm{CsCl}, \mathrm{AuCl}, \mathrm{ZnCl}_{2}\right)$. A huge advantage of salt flux is the simplicity of removal by solvent wash, especially by water. Owing to the lack of knowledge about elemental solubility in salts, the selecting of salt flux is an empirical process, until appropriate phase diagrams are made. An example of crystals of $\mathrm{CeCd}_{3} \mathrm{P}_{3}$ grown in $\mathrm{NaCl} / \mathrm{KCl}$ inert salt flux is shown in Figure 1D. Reactive fluxes often can be applied to overcome inertness of a transition metal. Thus, $\mathrm{BaT}_{2} \mathrm{P}_{4}$ and $\mathrm{BaT}_{2} \mathrm{P}_{3.75}(T=\mathrm{Cu}, \mathrm{Ni}, \mathrm{Au})$ can be produced starting from $\mathrm{TCl}$ or $\mathrm{TCl}_{2}$ precursors and excess of $\mathrm{Ba}$ (Kovnir et al., 2011; Fulmer et al., 2013a,b; Dolyniuk et al., 2015). Metal chlorides serve as source of $T$ element due to $\mathrm{Ba}+2 \mathrm{TCl} \rightarrow \mathrm{BaCl}_{2}+2 \mathrm{~T}$ reactions. Simultaneously, the mixture of barium and transition metal chlorides serves as a flux media.

TABLE 1 | Selected phosphide and arsenide crystals grown from flux.

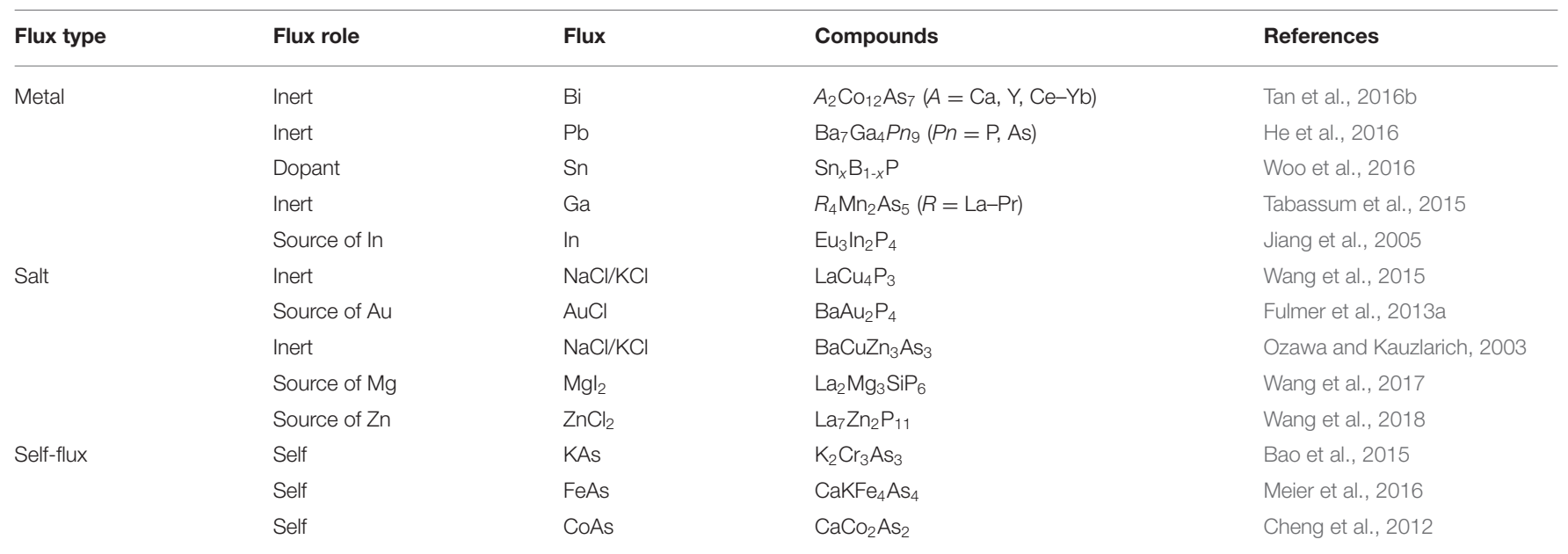


Finally, salt flux may be used to reduce the reactivity of a reactant. Metallic Cs is extremely reactive, challenging to handle, and requires a glovebox due to its pyrophoric nature. Instead, the $\mathrm{CsCl}+\mathrm{Mg}$ combination can be safely handled in air. $\mathrm{CsCl}$ is used as a flux and a source of $\mathrm{Cs}$ while producing a mixture of $\mathrm{Mg}$ and Cs chlorides that are removed by water washing after synthesis (Figure 1E) (Woo et al., 2019a).

\section{$\mathrm{La}_{2} \mathrm{Mg}_{3} \mathrm{SiP}_{6}$ in $\mathrm{Mgl}_{2}$ Flux}

As summarized in Table 1, salt flux was useful to grow compounds in $\mathrm{La}-M-\mathrm{P}$ systems $(M=\mathrm{Zn}, \mathrm{Cd}, \mathrm{Cu}, \mathrm{Mg}$, $\mathrm{Si}$ ). The crystal growth of $\mathrm{La}_{2} \mathrm{Mg}_{3} \mathrm{SiP}_{6}$ was impeded by the combination of inertness of $\mathrm{La}$ and $\mathrm{Si}$ and high reactivity and vapor pressures of $\mathrm{Mg}$ and $\mathrm{P}$ (Wang et al., 2017). Attempts to grow $\mathrm{La}_{2} \mathrm{Mg}_{3} \mathrm{SiP}_{6}$ using $\mathrm{Sn}$ or $\mathrm{Zn}$ metal flux were unsuccessful. Changing to $\mathrm{KCl} / \mathrm{NaCl}$ flux resulted only in a mixture of $\mathrm{Zn}_{3} \mathrm{P}_{2}$ and $\mathrm{SiP}_{2}$. Finally, $\mathrm{MgI}_{2}$ was proven to be proper flux for crystal growth of $\mathrm{La}_{2} \mathrm{Mg}_{3} \mathrm{SiP}_{6}$. Partial decomposition of $\mathrm{MgI}_{2}$ at reaction temperatures and release of iodine, which may serve as a transport agent might play a role in promoting reactivity of binary phosphides.

\section{Self-Flux}

The crystal growth by self-flux (flux composed solely of elements present in the target crystals) has the lowest chances of incorporating foreign elements. The self-flux is usually presynthesized, examples are provided in Table 1. When exploring the synthesis of a ternary system $\mathrm{ABC}$, all possible binary compounds, $\mathrm{AB}, \mathrm{AC}, \mathrm{BC}$ can be considered as self-flux if they meet the following criteria: facile synthesis, low melting and high boiling points, easy removal. For example, $\mathrm{CaAFe}_{4} \mathrm{As}_{4}$ ( $A=\mathrm{K}, \mathrm{Rb}, \mathrm{Cs})$ superconductors were initially discovered as polycrystalline samples, which only exist in a narrow temperature window (Iyo et al., 2016). Afterward, large crystals were grown from FeAs self-flux (Meier et al., 2016). A special crucible design, such as the Canfield crucibles, is very useful for separation of large crystals from flux (Canfield, 2020).

\section{Transport Growth in Flux}

Transport reactions in a liquid flux have a characteristic temperature gradient. At the hot end, reactants dissolve or form mobile species that migrate toward the cold end and crystallize in the target phase. This stands for endothermic equilibrium reaction, while for exothermic reaction, the transport happens from cold to hot end. Provided that the temperature gradient is consistent and includes the crystallization temperature of the target phase, crystal growth occurs on the cold end while simultaneously consuming reactants on the hot end, similar to the vapor transport reactions. Transport reactions do not require full solubility of reactants in the flux, allowing for a wider choice of fluxes and larger mass loadings of the starting materials, resulting in larger quantities of produced crystals. Transport reactions allow certain flexibility when selecting a flux because the solubility of reactants may not be as crucial. In turn, the viscosity and associated mass transport apply stricter constraints to the flux selection. This method is relatively new but has been demonstrated for crystal growth of antimonides, halides, chalcogenides, and transition metal chalcophosphates with $\mathrm{MPQ}_{3}(Q=\mathrm{S}$, Se) compositions (Yan et al., 2017). The development of transport reactions for crystal growth of phosphides and arsenides is currently underway. For liquid transport, presumably all of the fluxes previously discussed could allow crystal growth, which indicates that temperature gradients can be a useful variable when applied correctly. In a more in-depth review, Yan et al. (2017) demonstrated several examples of self-flux and salt flux used for transport growth.

\section{DISCUSSION AND PERSPECTIVE}

As discussed above, a researcher has several flux-based methods to grow single crystals of desired phosphides and arsenides. There is no universal, one-size-fits-all, flux method and no guarantee that for any given phosphide or arsenide the flux growth of cm-sized crystals can be developed. More systematic studies of flux growth mechanisms are required to make the latter statement false. Since the reaction and crystal growth are performed within a sealed environment to contain vapor pressure of pnictogens and prevent oxidation by air, the observation of reaction and crystal growth processes are limited. Many assumptions based on simple binary phase diagrams have to be made, which are not always correct, and not all binary phase diagrams are fully developed. Computational modeling of liquid-to-solid transformations in selected areas of complex phase diagrams may help reduce the number of trials for crystal growth. Recent developments of in situ techniques, TEM, and elastic scattering at synchrotron and neutron sources, revealed a lot of hidden processes occurring during flux synthesis, thus allowing for more rational design of flux reactions and crystal growth (Shoemaker et al., 2014; Woo et al., 2018, 2019b; Vasquez et al., 2019). Finally, computationally cheap methods, such as machine learning (Yao et al., 2019), can be applied to build bridges between "trial and error" and fully rational approaches. Results of unsuccessful trials, which are documented only in lab notebooks are required to train machine learning models and reveal hidden relationships.

Another challenge to be addressed is an adaptive control of crystal growth. Currently, most flux crystal growth relies upon spontaneous crystallization processes by manipulating temperature, initial reactants/flux ratios, and cooling rates. A design of a new type of flux crystal growth furnace, which can balance the inert atmosphere requirement and controlled growth with adaptive feedback loop, may provide larger and better quality crystals. The last to be mentioned, flux crystal growth under extreme conditions such as high temperature-high pressure remains essentially unexplored. Sparked by the recent discovery of almost room temperature superconductivity in $\mathrm{LaH}_{10}$ superhydride, there are rich opportunities to explore new phases and reaction mechanisms under applied pressure (Geballe et al., 2018). However, the challenges due to the 
presence of extreme conditions and necessity to work in limited sample environments are substantial. So far, crystals of superhydrides have not been produced and studied. Maybe high-temperature and high-pressure flux can help to grow crystals of superhydrides?

\section{AUTHOR CONTRIBUTIONS}

The manuscript was written through contributions of all authors. All authors have given approval to the final version of the manuscript.

\section{REFERENCES}

Babu, R., Giribabu, L., and Singh, S. P. (2018). Recent advances in halide-based perovskite crystals and their optoelectronic applications. Cryst. Growth Des. 18, 2645-2664. doi: 10.1021/acs.cgd.7b01767

Bao, J.-K., Liu, J.-Y., Ma, C.-W., Meng, Z.-H., Tang, Z.-T., Sun, Y.-L., et al. (2015). Superconductivity in Quasi-One-Dimensional $\mathrm{K}_{2} \mathrm{Cr}_{3} \mathrm{As}_{3}$ with significant electron correlations. Phys. Rev. X. 5:011013. doi: 10.1103/PhysRevX.5.011013

Bobev, S., Xia, S.-Q., Bauer, E. D., Ronning, F., Thompson, J. D., and Sarrao, J. L. (2009). Nickel deficiency in $\mathrm{RENi}_{2-\mathrm{x}} \mathrm{P}_{2}(\mathrm{RE}=\mathrm{La}, \mathrm{Ce}, \mathrm{Pr})$. Combined crystallographic and physical property studies. J. Solid State Chem. 182, 1473-1480. doi: 10.1016/j.jssc.2009.03.014

Bugaris, D. E., and zur Loye, H.-C. (2012). Materials discovery by flux crystal growth: quaternary and higher order oxides. Angew. Chem. Int. Ed. 51, 3780-3811. doi: 10.1002/anie.201102676

Canfield, P. C. (2020). New materials physics. Rep. Progr. Phys. 83:016501. doi: 10.1088/1361-6633/ab514b

Canfield, P. C., and Fisk, Z. (1991). Growth of single crystals from metallic fluxes. Philos. Mag. B. 65, 1117-1123. doi: 10.1080/13642819208215073

Cheng, B., Hu, B. F., Yuan, R. H., Dong, T., Fang, A. F., Chen, Z. G., et al. (2012). Field-induced spin-flop transitions in single-crystalline $\mathrm{CaCo}_{2} \mathrm{As}_{2}$. Phys. Rev. B 85:144426. doi: 10.1103/PhysRevB.85.144426

Coleman, N. Jr., Lovander, M. D., Leddy, J., and Gillan, E. G. (2019). Phosphorus-rich metal phosphides: direct and tin flux-assisted synthesis and evaluation as hydrogen evolution electrocatalysts. Inorg. Chem. 58, 5013-5024. doi: 10.1021/acs.inorgchem.9b00032

Dolyniuk, J., Wang, J., Lee, K., and Kovnir, K. (2015). Twisted Kelvin cells and truncated octahedral cages in the crystal structures of unconventional clathrates, $\mathrm{AM}_{2} \mathrm{P}_{4}(\mathrm{~A}=\mathrm{Sr}, \mathrm{Ba}, \mathrm{M}=\mathrm{Cu}, \mathrm{Ni})$. Chem. Mater. 27, 4476-4484. doi: 10.1021/acs.chemmater.5b01592

Dolyniuk, J., Zaikina, J. V., Kaseman, D. C., Sen, S., and Kovnir, K. (2017). Breaking the Tetra-Coordinated Framework Rule: New Clathrate $\mathrm{Ba}_{8} \mathrm{M}_{24} \mathrm{P}_{28+\delta}(\mathrm{M}=$ $\mathrm{Cu} / \mathrm{Zn})$. Angew. Chem. Int. Ed. 56, 2418-2422. doi: 10.1002/anie.201611510

Fulmer, J., Kaseman, D., Dolyniuk, J., Lee, K., Sen, S., and Kovnir, K. (2013a). $\mathrm{BaAu}_{2} \mathrm{P}_{4}$ : Layered Zintl Polyphosphide with Infinite $\left(\mathrm{P}^{-}\right)$Chains. Inorg. Chem. 52, 7061-7067. doi: $10.1021 /$ ic400584w

Fulmer, J., Lebedev, O. I., Roddatis, V. V., Kaseman, D., Sen, S., Dolyniuk, J., et al. (2013b). Clathrate $\mathrm{Ba}_{8} \mathrm{Au}_{16} \mathrm{P}_{30}$ : The "Gold Standard" for lattice thermal conductivity. J. Amer. Chem. Soc. 135, 12313-12323. doi: 10.1021/ja4052679

Geballe, Z. M., Liu, H., Mishra, A. K., Ahart, M., Somayazulu, M., Meng, Y., et al. (2018). Synthesis and stability of lanthanum superhydrides. Angew. Chem. Int. Ed. 57, 688-692. doi: 10.1002/anie.201709970

Gullman, J. (1990). The crystal structure of SnP. J. Solid State Chem. 87, 202-207. doi: 10.1016/0022-4596(90)90083-A

He, H., Stoyko, S., and Bobev, S. (2016). New insights into the application of the valence rules in Zintl phases - Crystal and electronic structures of $\mathrm{Ba}_{7} \mathrm{Ga}_{4} \mathrm{P}_{9}$, $\mathrm{Ba}_{7} \mathrm{Ga}_{4} \mathrm{As}_{9}, \mathrm{Ba}_{7} \mathrm{Al}_{4} \mathrm{Sb}_{9}, \mathrm{Ba}_{6} \mathrm{CaAl}_{4} \mathrm{Sb}_{9}$, and $\mathrm{Ba}_{6} \mathrm{CaGa}_{4} \mathrm{Sb}_{9}$. J. Solid State Chem. 236, 116-122. doi: 10.1016/j.jssc.2015.07.015

Iyo, A., Kawashima, K., Kinjo, T., Nishio, T., Ishida, S., Fujihisa, H., et al. (2016). New-Structure-Type Fe-Based Superconductors: $\mathrm{CaAFe}_{4} \mathrm{As}_{4}(\mathrm{~A}=$ $\mathrm{K}, \mathrm{Rb}, \mathrm{Cs})$ and $\mathrm{SrAFe}_{4} \mathrm{As}_{4}(\mathrm{~A}=\mathrm{Rb}, \mathrm{Cs})$. J. Am. Chem. Soc. 138:3410. doi: $10.1021 /$ jacs.5b12571

\section{FUNDING}

This work was supported by the Laboratory Research and Development Program of the Ames Laboratory under the U.S. Department of Energy Contract No. DE-AC02-07CH11358.

\section{ACKNOWLEDGMENTS}

JW was thankful for the support from Wichita State University start-up funds. PY was thankful to Chateaubriand Fellowship Program.

Jiang, J., Olmstead, M. M., Kauzlarich, S. M., Lee, H.-O., Klavins, P., and Fisk, Z. (2005). Negative magnetoresistance in a magnetic semiconducting Zintl phase: $\mathrm{Eu}_{3} \mathrm{In}_{2} \mathrm{P}_{4}$. Inorg. Chem. 44, 5322-5327. doi: 10.1021/ic0504036

Juillerat, C. A., Klepov, V. V., Morrison, G., Pace, K. A., and zur Loye, H.-C. (2019). Flux crystal growth: a versatile technique to reveal the crystal chemistry of complex uranium oxides. Dalton Trans. 48, 3162-3181. doi: 10.1039/C8DT04675A

Kanatzidis, M. G., Pöttgen, R., and Jeitschko, W. (2005). The Metal Flux: a preparative tool for the exploration of intermetallic compounds. Angew. Chem. Int. Ed. 44, 6996-7023. doi: 10.1002/anie.200462170

Kovnir, K., Garlea, V. O., Thompson, C. M., Zhou, H. D., Reiff, W. M., Ozarowski, A., et al. (2011b). Spin-glass behavior in $\mathrm{LaFe}_{x} \mathrm{Co}_{2-x} \mathrm{P}_{2}$ solid solutions: interplay between magnetic properties and crystal and electronic structures. Inorg. Chem. 50, 10274-10283. doi: 10.1021/ic201328y

Kovnir, K., Reiff, W. M., Menushenkov, A. P., Yaroslavtsev, A. A., Chernikov, R. V., and Shatruk, M. (2011a). Chemical metamagnetism": from antiferromagnetic $\mathrm{PrCo}_{2} \mathrm{P}_{2}$ to ferromagnetic $\mathrm{Pr}_{0.8} \mathrm{Eu}_{0.2} \mathrm{Co}_{2} \mathrm{P}_{2}$ via chemical compression. Chem. Mater. 23, 3021-3024. doi: 10.1021/cm200782z

Kovnir, K., Stockert, U., Budnyk, S., Prots, Y., Baitinger, M., Paschen, S., et al. (2011). Introducing a magnetic guest to a tetrel-free clathrate: synthesis, structure, and properties of $\mathrm{Eu}_{x} \mathrm{Ba}_{8-x} \mathrm{Cu}_{16} \mathrm{P}_{30}(0 \leq x \leq 1.5)$. Inorg. Chem. 50, 10387-10396. doi: 10.1021/ic201474h

Kovnir, K., Thompson, C. M., Garlea, V. O., Haskel, D., Polyanskii, A. A., Zhou, $\mathrm{H}$., et al. (2013). Modification of magnetic anisotropy through $3 \mathrm{~d}-4 \mathrm{f}$ coupling in $\mathrm{La}_{0.75} \mathrm{Pr}_{0.25} \mathrm{Co}_{2} \mathrm{P}_{2}$. Phys. Rev. B. 88:104429. doi: 10.1103/PhysRevB.88.104429

Kovnir, K., Thompson, C. M., Zhou, H. D., Wiebe, C. R., and Shatruk, M. (2010). Tuning ferro- and metamagnetic transitions in rare-earth cobalt phosphides $\mathrm{La}_{1-x} \mathrm{Pr}_{x} \mathrm{Co}_{2} \mathrm{P}_{2}$. Chem. Mater. 22, 1704-1713. doi: 10.1021/cm903497h

Lee, K., Synnestvedt, S., Bellard, M., and Kovnir, K. (2015). GeP and $\left(\mathrm{Ge}_{1-\mathrm{x}} \mathrm{Sn}_{\mathrm{x}}\right)\left(\mathrm{P}_{1-\mathrm{y}} \mathrm{Ge}_{\mathrm{y}}\right)(\mathrm{x}=0.12, \mathrm{y}=0.05)$ : Synthesis, structure, and properties of two-dimensional layered tetrel phosphides. J. Solid State Chem. 224, 62-70. doi: 10.1016/j.jssc.2014.04.021

Li, S., Zheng, Q., Lv, Y., Liu, X., Wang, X., Huang, P. Y., et al. (2018). High thermal conductivity in cubic boron arsenide crystals. Science 361, 579-581. doi: 10.1126/science.aat8982

Lindsay, L., Broido, D. A., and Reinecke, T. L. (2013). Firstprinciples determination of ultrahigh thermal conductivity of boron arsenide: a competitor for diamond? Phys. Rev. Lett. 111:025901. doi: 10.1103/PhysRevLett.111.025901

Liu, S., Joe, G., Zhao, H., Zhou, Y., Orvis, T., Huyan, H., et al. (2018). Giant optical anisotropy in a quasi-one-dimensional crystal. Nat. Photon. 12, 392-396. doi: 10.1038/s41566-018-0189-1

Mark, J., Wang, J., Wu, K., Lo, J. G., Lee, S., and Kovnir, K. (2019). Ba ${ }_{2} \mathrm{Si}_{3} \mathrm{P}_{6}$ : 1D nonlinear optical material with thermal barrier chains. J. Amer. Chem. Soc. 141, 11976-11983. doi: 10.1021/jacs.9b04653

Mathieu, J. L., and Latturner, S. E. (2009). Zintl phase as dopant source in the flux synthesis of $\mathrm{Ba}_{1-\mathrm{x}} \mathrm{K}_{\mathrm{x}} \mathrm{Fe}_{2} \mathrm{As}_{2}$ type superconductors. Chem. Commun. 33, 4965-4967. doi: 10.1039/b910749e

Meier, W. R., Kong, T., Kaluarachchi, U. S., Taufour, V., Jo, N. H., Drachuck, G., et al. (2016). Anisotropic thermodynamic and transport properties of single crystalline $\mathrm{CaKFe}_{4} \mathrm{As}_{4}$. Phys. Rev. B 94:064501. doi: 10.1103/PhysRevB.94.064501 
Nuss, J., Wedig, U., Xie, W., Yordanov, P., Bruin, J., Hübner,. R., et al. (2017). Phosphide-tetrahedrite $\mathrm{Ag}_{6} \mathrm{Ge}_{10} \mathrm{P}_{12}$ : thermoelectric performance of a long-forgotten silver-cluster compound. Chem. Mater. 29, 6956-6965. doi: 10.1021/acs.chemmater.7b02474

Owens-Baird, B., Kolen'ko, Y. V., and Kovnir, K. (2018). Structure-activity relationships for Pt-free metal phosphide hydrogen evolution electrocatalysts. Chem. Europ. J. 24, 7298-7311. doi: 10.1002/chem.201705322

Owens-Baird, B., Xu, J., Petrovykh, D. Y., Bondarchuk, O., Ziouani, Y., Gonzalez-Ballesteros, N., et al. (2019). $\mathrm{NiP}_{2}$ : a story of two divergent polymorphic multifunctional materials. Chem. Mater. 31, 3407-3418. doi: 10.1021/acs.chemmater.9b00565

Ozawa, T. C., and Kauzlarich, S. M. (2003). ( $\mathrm{NaCl}) /(\mathrm{KCl})$ flux single crystal growth and crystal structure of the new quaternary mixed-metal pnictide: $\mathrm{BaCuZn}_{3} \mathrm{As}_{3}$. Inorg. Chem. 42, 3183-3186. doi: 10.1021/ic034061k

Pamplin, R. B. (1980). Crystal Growth, 2nd Edition. Washington, DC: Pergamon Press.

Phelan, W. A., Menard, M. C., Kangas, M. J., McCandless, G. T., Drake, B. L., and Chan, J. Y. (2012). Adventures in crystal growth: synthesis and characterization of single crystals of complex intermetallic compounds. Chem. Mater. 24, 409-420. doi: 10.1021/cm2019873

Pöhls, J.-H., Faghaninia, A., Petretto, G., Aydemir, U., Ricci, F., Li, G., et al. (2017). Metal phosphides as potential thermoelectric materials. J. Mater. Chem. C 5, 12441-12456. doi: 10.1039/C7TC03948D

Saparov, B., and Bobev, S. (2010). Isolated infinite ${ }_{\infty}^{1}\left[\mathrm{ZnPn}_{2}\right]^{4-}$ chains in the Zintl phases $\mathrm{Ba}_{2} \mathrm{ZnPn}_{2}(\mathrm{Pn}=\mathrm{As}, \mathrm{Sb}, \mathrm{Bi})$ - Synthesis, structure, and bonding. Inorg. Chem. 49, 5173-5179. doi: 10.1021/ic100296x

Schäfer, K., Benndorf, C., Eckert, H., and, Pöttgen, R. (2014). PbP 7 - a polyphosphide with a three-dimensional $\left[\mathrm{P}_{7}\right]^{2--}$ network of condensed and P-bridged $\mathrm{P}_{6}$ hexagons. Dalton Trans. 43, 12706-12710. doi: 10.1039/C4DT01539H

Shatruk, M. (2019). "Synthesis of phosphides," in Fundamentals and Applications of Phosphorous Nanomaterials, ACS Symposium Series, ed H. Ji (Washington, DC: American Chemical Society), 103-134.

Shoemaker, D. P., Hu, Y.-J., Chung, D. Y., Halder, G. J., Chupas, P. J., Soderholm, L., et al. (2014). In situ studies of a platform for metastable inorganic crystal growth and materials discovery. Proc. Natl. Acad. Sci. U.S.A. 111, 10922-10927. doi: $10.1073 /$ pnas. 1406211111

Soheilnia, N., Xu, H., Zhang, H., Tritt, T. M., Swainson, I., and Kleinke, H. (2007). Thermoelectric properties of $\mathrm{Re}_{3} \mathrm{Ge}_{0.6} \mathrm{As}_{6.4}$ and $\mathrm{Re}_{3} \mathrm{GeAs}_{6}$ in comparison to $\mathrm{Mo}_{3} \mathrm{Sb}_{5.4} \mathrm{Te}_{1.6}$. Chem. Mater. 19, 4063-4068. doi: 10.1021/cm0708517

Tabassum, D., Lin, X., and Mar, A. (2015). Rare-earth manganese arsenides $\mathrm{RE}_{4} \mathrm{Mn}_{2} \mathrm{As}_{5}(\mathrm{RE}=\mathrm{La}-\mathrm{Pr})$. J. Alloys Compd. 636, 187-190. doi: 10.1016/j.jallcom.2015.02.145

Tan, X., Fabbris, G., Haskel, D., Yaroslavtsev, A. A., Cao, H., Thompson, C. M., et al. (2016a). A transition from localized to strongly correlated electron behavior and mixed valence driven by physical or chemical pressure in $\mathrm{ACo}_{2} \mathrm{As}_{2}(\mathrm{~A}=$ Eu, Ca). J. Amer. Chem. Soc. 138, 2724-2731. doi: 10.1021/jacs.5b12659

Tan, X., Garlea, V. O., Chai, P., Geondzhian, A. Y., Yaroslavtsev, A. A., Xin, Y., et al. (2016b). Synthesis, crystal structure, and magnetism of $\mathrm{A}_{2} \mathrm{Co}_{12} \mathrm{As}_{7}(\mathrm{~A}=\mathrm{Ca}, \mathrm{Y}$, Ce-Yb). J. Solid State Chem. 236, 147-158. doi: 10.1016/j.jssc.2015.08.038

Tan, X., Garlea, V. O., Kovnir, K., Thompson, C. M., Xu, T., Cao, H., et al. (2017). Complex magnetic phase diagram with multistep spin-flop transitions in $\mathrm{La}_{0.25} \mathrm{Pr}_{0.75} \mathrm{Co}_{2} \mathrm{P}_{2}$. Phys. Rev. B, 95:024428. doi: 10.1103/PhysRevB.95.024428

Tan, X., Tener, Z. P., and Shatruk, M. (2018). Correlating itinerant magnetism in $R \mathrm{Co}_{2} \mathrm{Pn}_{2}$ pnictides ( $R=\mathrm{La}, \mathrm{Ce}, \mathrm{Pr}, \mathrm{Nd}, \mathrm{Ca}, P n=\mathrm{P}, \mathrm{As}$ ) to their crystal and electronic structures. Acc. Chem. Res. 51, 230-239. doi: 10.1021/acs.accounts.7b00533

The National Academies of Sciences Engineering Medicine (2009). Frontiers in Crystalline Matter from Discovery to Technology. Washington, DC: The National Academies Press.

Thompson, C. M., Kovnir, K., Eveland, S., Herring, M. J., and Shatruk, M. (2011). Synthesis of $\mathrm{ThCr}_{2} \mathrm{Si}_{2}$-type arsenides from Bi flux. Chem. Commun. 47, 5563-5565. doi: 10.1039/clcc10578g
Thompson, C. M., Kovnir, K., Garlea, V. O., Choi, E. S., Zhou, H. D., and Shatruk, M. (2014a). Unconventional magnetism in $\mathrm{ThCr}_{2} \mathrm{Si}_{2}$-type phosphides, $\mathrm{La}_{1-x} \mathrm{Nd}_{x} \mathrm{Co}_{2} \mathrm{P}_{2}$. J. Mater. Chem. C. 2, 756-7569. doi: 10.1039/C4TC $00564 \mathrm{C}$

Thompson, C. M., Tan, X., Kovnir, K., Garlea, V. O., Gippius, A. A., Yaroslavtsev, A. A., et al. (2014b). Synthesis, structures, and magnetic properties of rare-earth cobalt arsenides, $\mathrm{RCo}_{2} \mathrm{As}_{2}$ ( $\left.\mathrm{R}=\mathrm{La}, \mathrm{Ce}, \mathrm{Pr}, \mathrm{Nd}\right)$. Chem. Mater. 26, 3825-3837. doi: $10.1021 / \mathrm{cm} 501522 \mathrm{v}$

Vasquez, G., Huq, A., and Latturner, S. E. (2019). In situ neutron diffraction studies of the metal flux growth of $\mathrm{Ba} / \mathrm{Yb} / \mathrm{Mg} / \mathrm{Si}$ intermetallics. Inorg. Chem. 58, 8111-8119. doi: 10.1021/acs.inorgchem.9b00857

Wang, J., Greenfield, J. T., and Kovnir, K. (2017). Synthesis, crystal structure, and magnetic properties of $\mathrm{R}_{2} \mathrm{Mg}_{3} \mathrm{SiPn}_{6}(\mathrm{R}=\mathrm{La}, \mathrm{Ce}, \mathrm{Pn}=\mathrm{P}, \mathrm{As})$. Inorg. Chem. 56, 8348-8354. doi: 10.1021/acs.inorgchem.7b01015

Wang, J., Lee, K., and Kovnir, K. (2015). Distorted phosphorus and copper square-planar layers in $\mathrm{LaCu}_{1+} \mathrm{P}_{2}$ and $\mathrm{LaCu}_{4} \mathrm{P}_{3}$ : synthesis, crystal structure, and physical properties. Inorg. Chem. 54, 890-897. doi: 10.1021/ic 502234w

Wang, J., Mark, J., Woo, K. E., Voyles, J., and Kovnir, K. (2019). Chemical flexibility of $\mathrm{mg}$ in pnictide materials: structure and properties diversity. Chem. Mater. 31, 8286-8300. doi: 10.1021/acs.chemmater.9b03740

Wang, J., Xia, S-Q., and Tao, X-T. (2013). Syntheses, crystal structure and physical properties of new Zintl phases $\mathrm{Ba}_{3} \mathrm{~T}_{2} \mathrm{As}_{4}(\mathrm{~T}=\mathrm{Zn}, \mathrm{Cd})$. J. Solid State Chem. 198 6-9. doi: 10.1016/j.jssc.2012.09.029

Wang, J., Yox, P., Voyles, J., and Kovnir, K. (2018). Synthesis, crystal structure, and properties of three La-Zn-P compounds with different dimensionalities of the Zn-P framework. Cryst. Growth Des. 18, 4076-4083. doi: 10.1021/acs.cgd.8b00445

Woo, K., Lee, K., and Kovnir, K. (2016). BP: synthesis and properties of boron phosphide. Mater. Res. Expr. 3:074003. doi: 10.1088/2053-1591/3/7/074003

Woo, K. E., Dolyniuk, J., and Kovnir, K. (2019a). Superseding van der Waals with electrostatic interactions: Intercalation of Cs into the interlayer space of $\mathrm{SiAs}_{2}$. Inorg. Chem. 58, 4997-5005. doi: 10.1021/acs.inorgchem.9b00017

Woo, K. E., Wang, J., Mark, J., and Kovnir, K. (2019b). Directing boronphosphorus bonds in crystalline solid: oxidative polymerization of $\mathrm{P}=\mathrm{B}=\mathrm{P}$ monomers into 1D chains. J. Amer. Chem. Soc. 141, 13017-13021. doi: 10.1021/jacs.9b06803

Woo, K. E., Wang, J., Wu, K., Lee, K., Dolyniuk, J.-A., Pan, S., et al. (2018). MgSi-As: an unexplored system with promising nonlinear optical properties. $A d v$. Funct. Mater. 28:180158. doi: 10.1002/adfm.201801589

Yan, J.-Q., Sales, B. C., Susner, M. A., and McGuire, M. A. (2017). Flux growth in a horizontal configuration: an analog to vapor transport growth. Phys. Rev. Mater. 1:023402. doi: 10.1103/PhysRevMaterials.1.023402

Yao, T.-S., Tang, C.-Y., Yao., Yang, M., Zhu, K.-J., Yan, D.-Y., Yi, C.-J., et al. (2019). Machine learning to instruct single crystal growth by flux method. Chin. Phys. Lett. 36:068101. doi: 10.1088/0256-307X/36/6/068101

Yu, T., Wang, S., Zhang, X., Li, C., Qiao, J., Jia, N., et al. (2019). MnSiP 2 : a new Mid-IR ternary phosphide with strong SHG effect and ultrabroad transparency range. Chem. Mater. 31, 2010-2018. doi: 10.1021/acs.chemmater.8b05015

Zavrazhnov, A. Y., Semenova, G. V., Proskurina, E. Y., and Sushkova, T. P. (2018). Phase diagram of the Sn-P system. J. Therm. Anal. Calorim 134, 475-481. doi: 10.1007/s10973-018-7123-0

Conflict of Interest: The authors declare that the research was conducted in the absence of any commercial or financial relationships that could be construed as a potential conflict of interest.

Copyright (c) 2020 Wang, Yox and Kovnir. This is an open-access article distributed under the terms of the Creative Commons Attribution License (CC BY). The use, distribution or reproduction in other forums is permitted, provided the original author(s) and the copyright owner(s) are credited and that the original publication in this journal is cited, in accordance with accepted academic practice. No use, distribution or reproduction is permitted which does not comply with these terms. 